\title{
SUNtool - A new modelling paradigm for simulating and optimising urban sustainability
}

\author{
D. Robinson ${ }^{\mathrm{a}, \mathrm{b}, *}$, N. Campbell ${ }^{\mathrm{a}}$, W. Gaiser ${ }^{\mathrm{a}}, \mathrm{K}$ Kabel $^{\mathrm{d}}$, A. Le-Mouel ${ }^{\mathrm{c}}$, N. Morel ${ }^{\mathrm{b}}$, \\ J. Page ${ }^{\mathrm{b}}$, S. Stankovic ${ }^{\mathrm{a}}$, A. Stone ${ }^{\mathrm{a}}$ \\ a BDSP Partnership Ltd, Summit House, 27 Sale Place, London W21 YR, UK \\ ${ }^{\mathrm{b}}$ LESO-PB, Station 18, Bâtiment LE, Ecole Polytechnique Fédérale de Lausanne, CH-1015, Switzerland \\ ${ }^{\mathrm{c}}$ Electricité de France, 77250 Moret-sur-Loing, France \\ ${ }^{\mathrm{d}}$ Faculty of Civil Engineering, Czech Technical University, Zikova 4, 166036 Prague 6, Czech Republic
}

Received 8 September 2006; received in revised form 5 March 2007; accepted 6 June 2007

Available online 16 July 2007

Communicated by: Associate Editor D. Yogi Goswami

\begin{abstract}
This paper describes the development and application of a new unique tool to support designers to optimise the sustainability of urban neighbourhoods (SUNtool). In this the paper introduces (i) the software architecture, (ii) the integrated solver and related innovations in the modelling of radiation exchange, reduced thermal modelling, stochastic modelling of occupant presence and behaviour, and urban plant modelling, (iii) interface design and innovations in building attribution, (iv) results analysis methods. Finally the software is applied to demonstrate its application to the development of urban planning guidelines and also to the design of a masterplan. (C) 2007 Elsevier Ltd. All rights reserved.
\end{abstract}

Keywords: Urban; Energy; Matter; Simulation; Optimisation; Software

\section{Introduction}

Urban neighbourhoods are complex entities. Buildings and other obstructions shade views from façades to the sky and sun, thus influencing radiant exchanges (shortwave, longwave and visual). These radiant exchanges, together with anthropogenic gains, evapotranspiration losses and the production/dissipation of turbulent kinetic energy influence the hydrothermal microclimate (temperature, velocity and air pressure). This urban microclimate influences pedestrian comfort, building energy demands and to a degree irrigation water demands. A further influence on building energy demands, besides the inherent

\footnotetext{
* Corresponding author. Address: LESO-PB, Station 18, Bâtiment LE, Ecole Polytechnique Fédérale de Lausanne, CH-1015, Switzerland. Tel.: +41 21693 4543; fax: +41216932722.

E-mail address: darren.robinson@epfl.ch (D. Robinson).
}

characteristics of the buildings themselves, is the behaviour of the occupants accommodated. The temporal characteristics of presence as well as interactions with the appliances, fabric and systems is stochastic in nature, so that even identical buildings will have different temporal profiles for the demands for applied energy, the consumption of water and the production of waste water and refuse. This has important implications on the aggregate flows of urban resources. On a related note, it is also possible to benefit from synergetic exchanges between buildings (e.g. heat rejection from one as a source for another) and between resources (e.g. the derivation of energy from waste). Finally, due to differences in individual building resource flows it is possible to specify decentralised plant which is smaller than the sum of the required individual capacities. This, together with economies of scale, makes the decentralised management of resources attractive, with associated environmental dividends. 
An EC funded research project SUNtool (sustainable urban neighbourhood modelling tool) was conceived to address this urban modelling complexity, in order to assist in the design of more sustainable urban neighbourhoods (composed of say 50-500 buildings) based on accurate simulations of resource (energy, water and waste) flows. In this the software should enable the designer to optimise the layout, form and fabric design of buildings to minimise energy demands as well as to choose the optimum combination of technologies to supply and control energy and to process water and waste - all accounting for sensitivities to microclimate and human behaviour. This implies the development of an early decision making tool for sustainable urban design.

At face value, this objective seems like something of a contradiction in terms - not only is greater modelling capability being introduced than is handled in single-building simulation programs, but many buildings are proposed to be modelled. This potentially increases not only the data requirements but also the complexity of results interpretation to arrive at meaningful decisions. Aside from addressing the modelling challenges mentioned above, a further objective was therefore to develop an interface to the models which is quick and intuitive to use - both for description and results interpretation. A final objective is that the results should be available within a reasonably short period of time, to usefully inform early master planning decisions.

This paper describes how each of these objectives has been realised. First we give an overview of the software design and proceed to describe in detail the approach to interface development - addressing geometry definition, attribution, calculation control and analysis. We then describe the structure of the solver and related models, before briefly discussing some software integration issues. After presenting examples of the application of the SUNtool software to some case study problems, we conclude by discussing some current limitations and future prospects for the software.

\section{Overview of software architecture}

The overall conceptual structure of the software (see also Robinson et al., 2003) is illustrated in Fig. 1. In brief, the user selects the global location of the site using the user interface (developed in Java) and this in turn selects climate data and an iDefaults (or intelligent defaults) dataset. This latter contains detailed attribution information for buildings as a function of age and type of use/occupancy. The user proceeds to define the geometry of each building, with Microsoft DirectX used as the rendering engine for Java3D. Default building uses may then be overridden and indeed buildings may be split into several uses. Detailed characteristics defining these buildings/uses may then be tailored to the particular case under examination. Likewise default building-embedded HVAC system descriptions may be refined. Additional plant items, including renewable energy and water processing technologies,

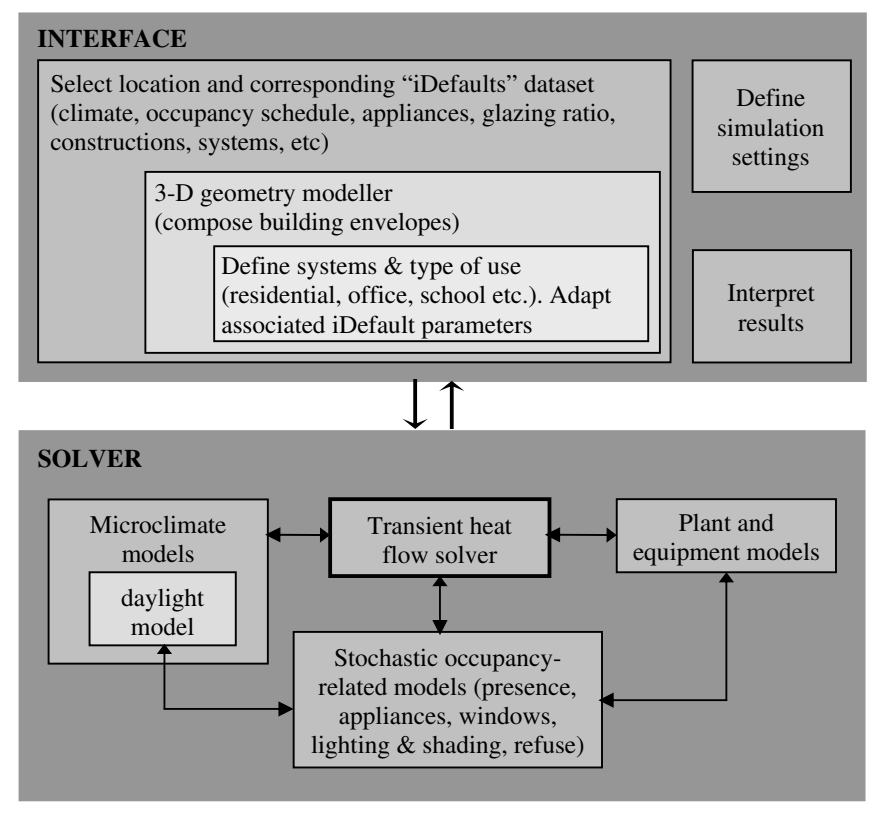

Fig. 1. Conceptual design of the SUNtool software.

may also be selected, whether embedded or contained within a central energy centre. Simulation settings are defined. Options include one-off annual simulations (batches of these may also be defined) or parametric simulations, in which particular parameters are varied between lower and upper bounds according to some specified increment. Subsets of a potential solution may also be selected. For example, we may simulate the microclimate or energy flows only; possibly for a particular time period and/or selection of the described buildings.

The model details and simulation settings are then parsed, via XML files, to the solver. A 'scene factory' creates and populates the relevant $\mathrm{C}++$ objects which are then handed to an instance of the solver. An instance of each model (microclimate, thermal, stochastic and plant) is then created. Zone objects (buildings or parts of) store state information relating to individual models, so that data for internal model communication is held in memory.

Results for each simulation time step are continuously streamed back to the interface, in the form of an XML document.

\section{Solver design}

As indicated in Fig. 1, there are four key classes of model: ${ }^{1}$ Microclimate, Thermal, Stochastic and Plant. For each class a review of relevant modelling techniques was conducted. From this, modelling methods were selected or adapted based on their fitness for purpose. The test here was that their degree of complexity should

\footnotetext{
${ }^{1}$ Although not described here, there is also a set of associated modelling functions, related to internal zoning of buildings, water reclamation, resource use due to external activities such as street lighting, etc.
} 
be compatible with that of other models as well as with the quantity of data available to populate them and with the requirement for rapid simulation time whilst achieving predictions of acceptable accuracy. The outcomes in each case are described below.

\subsection{Microclimate models}

Relationships between urban form and microclimate were introduced in Section 1 and these are discussed, along with associated modelling approaches, in further detail in Robinson (2006). In principle it would be useful to have a comprehensive hydrodynamic model simulating the velocity, temperature and pressure field throughout our neighbourhood, influenced by radiative processes (short and longwave exchange), anthropogenic sources and evaporative sinks, all with due regard for the influences of the bounding urban structure (including the heat island effect). However, this is not computationally tractable within the present context. Our more modest aims were firstly to account for the effects of obstructions on shading views to the sky and sun and contributing reflected radiation for predictions of radiation exchanged at building envelopes. Our second aim was to account for the temperature differences between rural weather stations at which our climate data is measured and our local urban context.

\subsubsection{Radiation modelling}

Considering for the moment just shortwave radiation exchange our objective is, at a given point on an urban surface, to predict the incident irradiance accounting for obstructions to the sky, the sun and also for the contribution of reflected radiation. At the start of this project, only general radiosity or ray tracing methods could account for these processes, but the computational costs are excessive recall that this is just one part of an integrated solver addressing potentially hundreds of buildings each comprised of perhaps tens of surfaces. An alternative is to adapt simplified tilted surface irradiance models to account for urban obstructions by representing adjacent obstructions in the form of a continuous canyon. In fact an approximation of this approach was attempted by Steemers (1992) in conjunction within an isotropic sky model. On this basis, a mathematically more rigorous implementation, applied to isotropic and anisotropic sky models was developed (Robinson and Stone, 2004), but even this was found to lead to significant errors as the geometric integrity of the problem is not retained and because the sky anisotropy is either ignored or focussed into a horizon belt and a circumsolar point. Our solution was to take the principal of radiosity modelling and include a series of simplifications.

For some set of $p$ sky patches, each of which subtends a solid angle $\Phi(\mathrm{Sr})$ and has radiance $R\left(\mathrm{~W} \mathrm{~m}^{-2} \mathrm{Sr}^{-1}\right)$ then, given the mean angle of incidence $\xi(\mathrm{rad})$ between the patch and our receiving plane together with the proportion of the patch that can be seen $\sigma(0 \leqslant \sigma \leqslant 1)$, we have a general solution (Robinson and Stone, 2004) for direct sky irradi- ance $I_{d \beta}\left(\mathrm{W} \mathrm{m}^{-2}\right): I_{d \beta}=\sum_{i=1}^{p}(R \Phi \sigma \cos \xi)_{i}$ and reflected irradiance may be similarly calculated.

This is implemented as follows:

- Within an urban scene, a given surface may experience considerable variability in terms of sky view (i.e. we have spatial variation in $\sigma$ ). A grid is thus imposed on each surface and a low resolution narrow view $\left( \pm 45^{\circ}\right.$ hoz/vert) rendering at each cell is processed to determine the fraction of unobstructed sky that is visible. These surfaces are then split (horizontally of vertically) where thresholds for the gradient in sky view factor are exceeded (Fig. 2). The algorithm then proceeds to calculate the irradiance at the centroid of these new smaller surfaces.

- Views encapsulating the hemisphere are rendered from each (new) surface centroid, with every surface having a unique colour. Each pixel is translated into angular coordinates to identify the corresponding patch as well as the angle of incidence. For sky view factors then, $\Phi \sigma \cos \xi$ is treated as a single quantity obtained by numerical integration of $\cos \xi \cdot \mathrm{d} \Phi$ across each sky patch. Likewise for $\Phi \omega \cos \xi$, where $\omega$ is the patch occlusion view factor. This latter is used to calculate the reflected irradiance $I_{\rho \beta}=\sum_{i=1}^{2 p}\left(R^{*} \Phi \omega \cos \xi\right)_{i}$ in which $R^{*}$ is the radiance of the dominant surface, identified (from its colour) as that which occludes the greatest proportion of the $i$ th patch. ${ }^{2}$

- A similar process is repeated for solar visibility fractions for each surface, for which a constant size scene is rendered from the sun position. The ratio of the number of pixels that can be seen of each surface to those that would be visible if each surface was unobstructed defines the visibility fraction $\sigma$ for time $t$ so that the incident beam irradiance $I_{b \xi}$ is $I_{b n} \sigma_{t} \cos \xi$.

- A set of simultaneous equations relating the beam and diffuse sky components to each surface's irradiance, which itself effects the reflected irradiance incident at other surfaces, is formulated as a matrix and solved iteratively until a convergence criterion is passed.

External surface illuminance may be solved in a similar way, given the solar illuminance and a sky luminance distribution. Views to the external sources may then be determined from renderings taken at internal points to calculate the sky and externally reflected contributions to internal illuminance (Robinson and Stone, 2006). These are also inputs to a low resolution conventional radiosity calculation (Robinson and Stone, 2005a) to solve for internally reflected contribution to internal illumination in an accurate but fast way.

The view parameters may also be used to predict external longwave radiation exchange in a simplified way and

\footnotetext{
${ }^{2}$ Note that this is summed over $2 p$ patches, because an inverted dome is used to account for reflected radiation below the horizontal plane.
} 

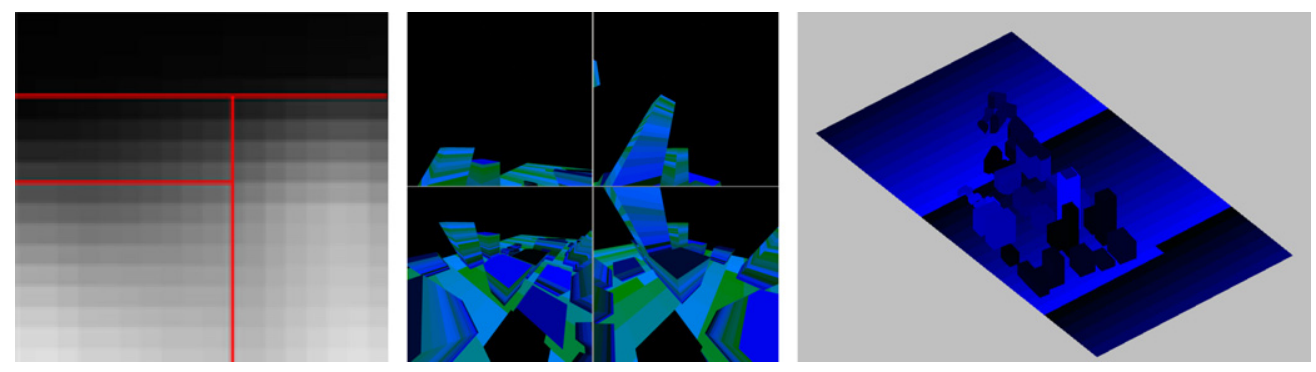

Fig. 2. Splitting of surface based on calculations sky view proportion using renderings from surface grid cells (left), renderings from new surface centroids for calculation of patch and obstruction view factors - and associated surface identification (centre), renderings from sun position for calculation of sun visibility fraction.

the set of the radiant exchanges (shortwave, visible and longwave) can be solved within a single computational module: see Robinson and Stone (2005a) for a more comprehensive description of this simplified radiosity algorithm (SRA).

Comparisons of predictions of shortwave and visible radiation exchange with the backwards Monte Carlo ray tracing program RADIANCE (Ward Larsen and Shakespeare, 1997) show (Fig. 3) that similarly accurate results are achieved at a computational cost at least five orders of magnitude lower. Note that results from detailed comparisons of hourly predictions from the SRA with RADIANCE at a specific point in an urban scene are presented in Robinson and Stone (2004).

Finally, Robinson and Stone (2005b) describe how the SRA can be incorporated into standard building simulation programs (i.e. to solve for general building/room geometries).

\subsubsection{Temperature prediction}

It is recognised by the authors that a proper approach to the modelling of the urban heat island effect should consider each of the key physical process which influences it. Several mesoscale atmospheric flow models incorporate urban parameterisation schemes to estimate the heat, momentum and turbulent fluxes within the urban canopy layer (Masson, 2000; Kusaka et al., 2001; Martilli et al., 2002a), albeit based on simple repeated building and street geometries. Indeed Robinson (2006) suggests that a onedirectional coupling between such models and a simplified CFD model within a single mesoscale grid cell could account for large scale hydrothermal effects in producing locally meaningful predictions of velocity, temperature and pressure at specific building surfaces. However, the resources required to develop such a modelling capability (in computationally tractable form) were not available to this project. Therefore, considerably more simplified models were reviewed which would give rapid order-of-magnitude predictions of local temperature.

Oke (1973) has suggested a relationship between urbanrural temperature differences and city population (as a surrogate for size) and wind speed (having a diluting influence), but this does not account for urban albedo and associated consequences for short and long wave radiation exchange, nor for evapotranspiration sinks and anthropogenic sources. It also does not account for position within a city (the epicentre of the heat island has been observed to occur close to the city centroid, unless deflected by wind (Graves et al., 2001). In later work, Oke (1988) related street canyon proportions to maximum heat island intensity, but this now implies that intensity is independent of city size. It also does not deal explicitly with the sources

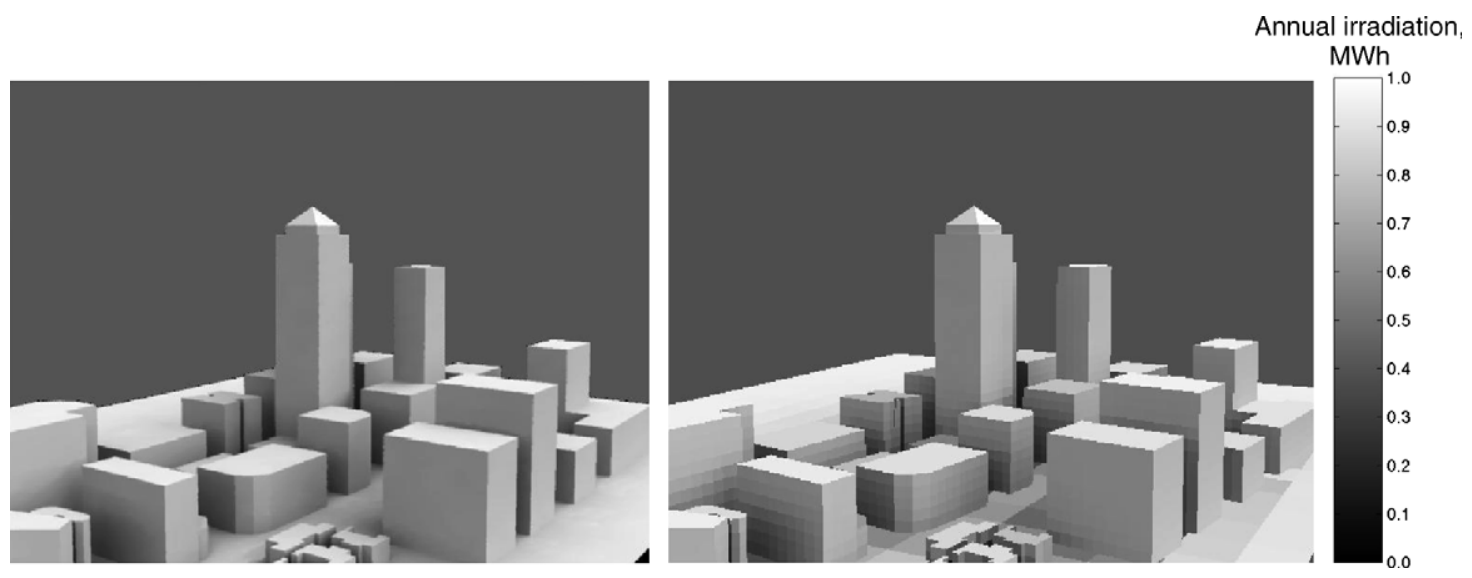

Fig. 3. Predictions of annual solar irradiation throughout a simplified 3D model of Canary Wharf (London, UK) from RADIANCE (left) and the SRA (right) - based on surfaces of $\leqslant 10 \mathrm{~m} \times 10 \mathrm{~m}$. 
and sinks mentioned above, nor with positional variations (the result relates to the centroid).

Some of the weaknesses in these empirical expressions are in principal resolved by the physical model due to Swaid (1988) and Swaid and Hoffman (1989, 1990a,b) and later amended by Elnahas and Williamson (1997). This model assumes that individual urban surfaces thermophysical characteristics can be represented by a single Cluster Thermal Time Constant (CTTC) to determine an exponential response in temperature to changes in surface flux. ${ }^{3}$ Several improvements to the CTTC model were tested:

- Use of the SRA for an accurate calculation of shortwave radiation exchange and the associated thermal response.

- Exponential response to longwave radiation exchange.

- A continuous rather than discrete solution to the exponential response function to surface flux changes.

However, even with these improvements and by using numerical methods to optimise certain of the model coefficients, it was not possible to produce convincing predictions of urban temperature profiles (it was not even possible to produce convincing reproductions of a known rural temperature profile using the Swaid and Hoffmann formulation).

Following this work it was decided that the uncertainties in predictions from these simplified models may be similar or larger in magnitude than the errors due to ignoring urban-rural temperature differences, so that no thermal microclimate model is included in SUNtool for the present time.

\subsection{Thermal modelling}

The prediction of thermal energy demands for space heating and cooling (as well as indoor temperature) is relatively well understood - myriad models have been developed at various degrees of detail, ranging from steady state, through cyclic, to explicit dynamic predictions using response function or finite difference techniques (Clarke, 2001 ). As with other models, our approach within this project is to achieve reasonably accurate hourly predictions of thermal energy flows in a way that is appropriate to the level of knowledge available describing our system (the buildings) in a computationally efficient way.

Following a review of simplified thermal modelling techniques, the decision was taken to adopt the grey-box approach due to Déqué et al. (2000).

The energy balance on a building envelope may be represented as: $\rho V C_{p} \frac{\partial T}{\partial t}=\sum_{i=1}^{n} \varphi_{i}$ with $\rho V C_{p}$ being the thermal capacitance, $T$ the indoor temperature and $\varphi_{i}$ is either an energy flux across a building element or an internal gain (e.g. due to people, lights or appliances). This may be

\footnotetext{
${ }^{3}$ In fact, an exponential response to shortwave radiation only was considered in these versions of CTTC, on the assumption that longwave exchange is relatively constant throughout the day.
}

re-expressed as a "state equation" on the basis of a spatial discretisation of physical laws, so that:

$$
\begin{aligned}
& T^{\prime}=A T+B I \\
& T_{\text {air }}=C T
\end{aligned}
$$

Here $I$ is an input vector, $T_{\text {air }}$ is the internal zone temperature, $T$ is a state vector and $T^{\prime}$ is its derivative $(\mathrm{d} T /$ $\mathrm{d} t) . A$ is the state matrix, $B$ the order matrix and $C$ the observation matrix.

A "white box" model (an explicit dynamic simulation program) is used to produce the state equation representation of the system, as defined above. This model is then reduced using Moore's truncation technique (Moore, 1981), which involves retaining only the most controllable and observable state ${ }^{4}$ components of the system. The resultant "black-box" model faithfully reproduces (both stationary and transient predictions of) the physical model, but lacks physical meaning. In a final step, the main thermal and geometric parameters of the physical model are connected to the reduced model, firstly based on conservation of static and then on transient gains. In this the state equation is transformed into a "transfer function" representation.

For SUNtool, the zone air temperature is related to four inputs:

- heating or cooling energy delivered to or generated within the zone (air node),

- solar flux transmitted through fenestration into the zone,

- solar flux absorbed within the zone,

- external or ambient temperature.

each being described by a separate transfer function of the form:

$$
T_{\text {air }}(p)=\sum_{i=1}^{4} F_{i}(p) \times I_{i}(p)
$$

where $I_{i}(p)$ are the inputs of the model, $T_{\text {air }}(p)$ is the resulting zone air temperature and $F_{i}(p)$ are the second order transfer functions:

$F_{i}(p)=\frac{1}{H_{i}} \times \frac{\left(1+\mathrm{TN} 1_{i} \times p\right) \times\left(1+\mathrm{TN} 2_{i} \times p\right)}{(1+\mathrm{TD} 1 \times p) \times(1+\mathrm{TD} 2 \times p)}$

with $H_{i}$ being the static gain of the transfer function, $\mathrm{TN}_{i}$ and $\mathrm{TN} 2{ }_{i}$ are "equivalent" time constants in the numerator, TD1 and TD2 are time constants in the denominator and $p$ is the derivative operator: so that $p * F_{i}(p)$ is the transform of $\mathrm{d} F_{i}(t) / \mathrm{d} t$.

In these transfer functions the static gain $H_{i}$ expresses the relation between the input and output under steadystate conditions (with a unique value derived for each

\footnotetext{
${ }^{4}$ Controllability may be defined as the possibility to obtain and vary the model states using the system inputs, whereas observability is the possibility to determine the model states from the outputs.
} 
input). $\mathrm{TNX}_{i}$ represents the dynamic response of the output to each (unit) variation in the input (again unique for each input). The time constants in the denominator are related to the building (i.e. constant for all inputs), with TD1 being the main time constant for the whole building, in effect a measure of its thermal inertia.

This model is implemented in a two step predictor-corrector form. Initially the set point temperature is used to solve for the associated energy demands. If the energy available is less than required then the consequent indoor air temperature is obtained in a second pass.

Although model verification using the BESTEST methodology (Judkoff and Neymark, 1995) has shown encouraging results, it should be noted that this grey-box modelling approach is intrinsically limited to the cases from which the inputs to the transfer functions are defined (e.g. cases with roof glazing or atria are not well modelled).

\subsection{Stochastic modelling}

Humans are intrinsically unpredictable animals. Moreover, their presence and interactions have important implications for a building's energy balance, affecting both the indoor microclimate and the demands for applied energy.

People enter a building, leave it and move within it in stochastic ways - albeit informed by common practice for arrival, departure and key breaks. They may also engage in overtime, they may fall ill and they may take vacations - again these are stochastic processes. The presence of people within a thermal zone leads directly to the emission of pollutants and metabolic heat gains. Clearly, human interactions which influence the energy balance also depend on presence. Examples include interactions with:

(i) window and door openings: influencing air flow,

(ii) shading devices/blinds: influencing radiation transmission and glass surface temperature,

(iii) lighting controls: influencing electricity consumption and casual heat gains,

(iv) electrical appliances: influencing electricity consumption and casual heat gains,

(v) heating, ventilating and cooling system controls: influencing thermal and electrical energy consumption and associated heat injection/rejection.

Waste is also produced, from which energy may be derived, and water is consumed.

Yet both the presence of people and these interactions tend to be handled in entirely deterministic ways (if at all) in current building simulation programs. People are assumed to arrive and leave according to some perfect and repeated time schedule. If ventilation openings are controlled this is likely to be based on either a time schedule, with no environmental stimulus, or due to an environmental stimulus that is repeated (e.g. always have the window open while the air temperature is above $25^{\circ} \mathrm{C}$ ). Shading devices, if controlled (they are normally not), react in a perfect and repeated way according to some physical stimulus such as transmitted solar radiation. Lighting control is similar, but based on some illuminance threshold [which itself may be derived from a daylight factor, or based on links with detailed and computationally expensive lighting simulation software]. Electrical appliances are normally operated according to some repeated time schedule, often coinciding with occupant presence, and at some average intensity (e.g. based on average power demand). Finally, HVAC plant is normally assumed only to be automatically controlled according to fixed set points. In reality these controls are frequently overridden and, where the control exists, set points may vary both spatially (in different parts of the same building) and with time.

The key problems with populating our virtual buildings with these virtual robots are twofold. Firstly, in poorly solving for the thermal energy balance, we have corresponding errors both regarding the indoor environmental conditions in the free-floating case and in the energy demands in maintaining pre-set conditions. Secondly, and of increasing importance, is that the aggregate thermal and particularly electrical demand profile, due to multiple buildings, may be seriously in error - these profiles, particularly the peaks, tend to be asynchronous. Consequently, the plant requirements for installations such as district co-generation may also be seriously in error. Likewise for the predicted energy consumption. Both affect the economic feasibility of locally centralised energy generation proposals.

Robinson (2006) presents a brief review of developments in stochastic modelling relating to each of the categories defined above. Here we briefly describe the approaches adopted within SUNtool. Note that no attempt has yet been made to predict interactions with HVAC systems.

\subsubsection{Occupant presence}

The occupancy model is described in detail, along with results from extensive validation studies, by Page et al. (submitted for publication). The model is derived from two key hypotheses: (i) that actions at the current time step depend only upon the status of the inputs at the previous time step, and (ii) that all occupants are independent from one another. More specifically:

- Using quarter-hourly profiles (the model is temporally discretised) of occupancy probability (which may be different for each day type), and results from the previous time step together with a 'mobility' parameter, the probability of occupancy at the present time step is determined, based on the theory of Markov Chains.

- The probability that a long absence will start is also determined, to account for vacations or possibly business trips or sickness, in the case of offices. The duration is randomly selected, following a cumulative probability distribution which can be freely defined by the SUNtool user (a default distribution is available in the iDefaults). During this long absence, the above calculation is disabled for the occupant affected. 
- The thermal consequences of this and related models are aggregated, producing hourly loads which are parsed to the building thermal model.

Results from comparisons between measured and simulated data for singly occupied offices are shown in Figs. 4a and $4 \mathrm{~b}$. These indicate that key trends relating for example to times of arrival and cumulative periods of presence are well reproduced by the model - tending to confirm the first hypothesis stated above. The second hypothesis in principal prevents us from simulating cases in which the presence of multiple persons within a zone depend upon the presence of some key person (or event). However, this can be accommodated by multiplying the profile for this key person by the number of people effected (which may be a deterministic or a stochastic variable).

\subsubsection{Window openings}

An attempt has been made to develop a behavioural model of window openings, relating real indoor stimuli with interaction probability. In this there are two aspects to the model: the prediction of the probability of interaction, and the consequences of this interaction.

Two types of stimuli are accounted for in predicting interaction probability - indoor temperature ${ }^{5}$ and pollutant concentration, so that the effects of opening windows is to dilute internal pollutants or to introduce cooler outside air.

From the work of Fanger (1988), it is possible to predict internal pollutant concentration, given an outside concentration and a known source of internal pollution. Furthermore, by inverting the equation proposed by Fanger to relate the percentage of people dissatisfied (PPD), with indoor pollutant concentration, one can determine some personal (that is individual) critical pollutant concentration (beyond which interaction will take place) $C^{*}$, based on some randomly chosen limiting PPD:

$C^{*}=(\ln [\mathrm{PPD} / 395] / 3.25)^{-4}$

In our case we distinguish between the acceptability of people already present in a zone and that of new arrivals, who are presumed to have a lower tolerance (they are more sensitive to pollution than those that have become accustomed to a progressive build up). Interaction takes place when the resultant calculated indoor concentration $\left(C_{i}\right)$ exceeds the critical concentration $C^{*}$.

Also based on the work of Fanger, the ISO7730 comfort model is used to calculate the air temperatures which correspond to $\mathrm{PMV} \pm 2$, chosen to be critically hot or cold comfort limits for a population of people. A temperature increment $(\Delta T)$ is then randomly selected, from a normal distribution with a mean of zero and standard deviation of $2{ }^{\circ} \mathrm{C}$. New personal critical limits, with this random

\footnotetext{
${ }^{5}$ In contrast to the work of Nicol (2001) and Herkel et al. (2005), which relate interactions to outdoor stimuli, so that these actions are independent of the internal situation (and thus of the design of the building itself), we produce building-dependent predictions.
}
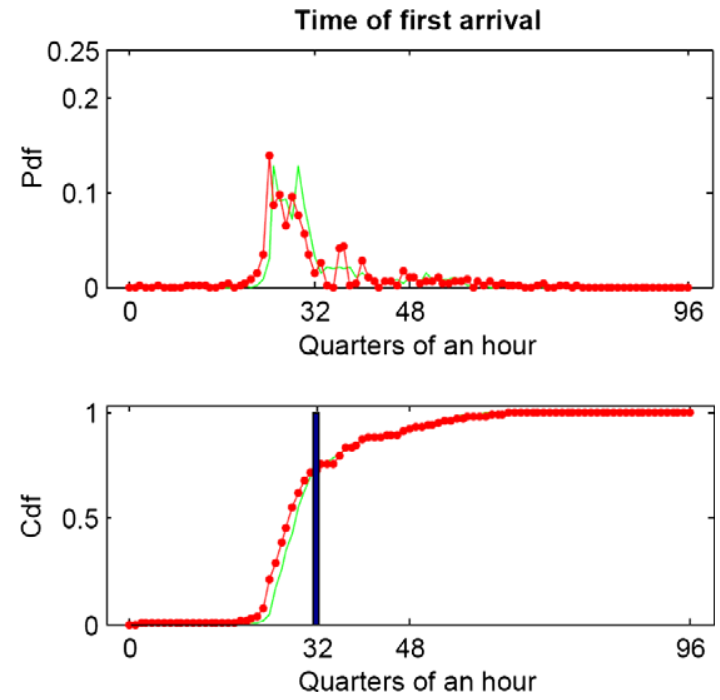

Fig. 4a. Measured (green) and simulated (dotted red) distributions of time of arrival - quarters of an hour from midnight. The blue vertical bar represents a standard assumption ( 8 a.m. arrival).
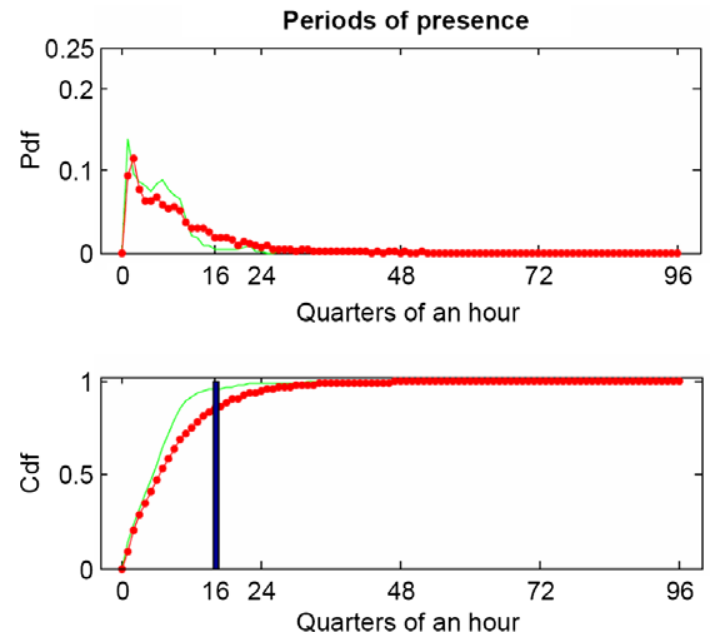

Fig. 4b. Measured (green) and simulated (dotted red) distributions of cumulative periods of presence - quarters of an hour. The blue vertical bar represents a standard assumption $(8 \mathrm{~h} /$ day $)$.

component, are now calculated $\left(T_{\text {hot }}=T_{\text {hot(ave) }}+\Delta T\right.$; $\left.T_{\text {cold }}=T_{\text {cold(ave) }}+\Delta T\right)^{6}$ and compared with the current temperature to decide whether windows would be shut ( $\left.T<T_{\text {cold }}\right)$ or opened $\left(T>T_{\text {hot }}\right)$. Note that there is no such pollutant stimulus causing occupants to close the window, which occupants are assumed to do (during the heating season) if they are about to leave for a long period of time.

The consequence of the interaction is that the opening of a window induces a deterministically calculated ventilation

\footnotetext{
${ }^{6} T_{\text {hot(ave) }}, T_{\text {cold(ave) }}$ correspond to the critical limits of an average population whereas the increments account for individual variances within this population.
} 
rate. Given the lack of knowledge regarding the internal layout of the building and difficulties in determining surface pressure coefficients in urban areas, the ventilation rate is simply calculated assuming single-sided buoyancy driven ventilation. Additionally, given the low specific heat capacity of air, it is useful to associated this simple ventilation model with a thermal model which has a shorter time step. Otherwise windows may be open/closed for an unrealistically long period of time, causing corresponding errors in heat and pollution exchange. For this a separate resistance-capacitance thermal model is used to calculate the indoor temperature using a time step of $5 \mathrm{~min}$. This process is repeated during the hourly time step to determine the mean advective heat transfer due to changes in opening status and internal buoyancy pressure. This result is then parsed to the whole building thermal model. The initial air temperature of the simplified thermal model model is re-seeded using results from the whole-building thermal model at the start of each hourly time step.

We assume that the deterministic aspects of the models of interaction stimulus described above, having been based on empirically derived models which have subsequently become enshrined in international standard, are reasonable. ${ }^{7}$ Data to validate the stochastic models of interaction stimuli on the other hand has not been obtained, although plausibility checks have been made.

\subsubsection{Lights and shading devices}

The control of artificial lighting has direct consequences for electrical energy consumption and this and interactions with blinds/shading devices have indirect consequences for space conditioning energy demand. The model for interactions with lighting and blinds in this project is based on Reinhart's (2004) tool Lightswitch-2002. This accounts for stochastic light switching at arrival and during occupancy as a function of minimum indoor illuminance as well as at departure, based either on prior knowledge of the duration of this event (manual operation) or on automatic PIR control. The models of each of these types of interaction have been individually validated. Lightswitch-2002 also accounts for the probability of opening blinds at arrival and of interacting with them as a function of glare risk. This latter though is somewhat crude, being based only on some threshold solar irradiance incident on the window plane.

In SUNtool the blind position is first evaluated for the current time step, so that the implications for natural internal illuminance can be determined. The daylight model then calculates an illuminance based on the appropriate transmittance for the region of window seen from each of

\footnotetext{
${ }^{7}$ It is acknowledged however that, due largely to a lack of consideration of human adaptation to discomforting events, the accuracy of the Fanger thermal comfort model is questionable for free-floating environments. However, a complete adaptive model has until now proven elusive. Instead, we may consider that the stochastic definition of personal comfort thresholds is a partial response to such adaptive processes.
}

two calculation points (one at the front and one at the back of the room), to account for partially lowered roller blinds. Depending upon occupant presence this may then provoke a light switching action.

A future implementation might include blind utilisation factors in the lighting model, to account for more complex types of blind (e.g. the redirection of light from a louvre blind).

\subsubsection{Electrical and water appliances}

In SUNtool the general concept 'appliance' includes all electricity and/or water consuming devices. These appliances are individually represented within each zone of each building. Each appliance is allocated to one of four categories:

- Category A: use of the appliance is independent of occupancy (e.g. a refrigerator ${ }^{8}$ );

- Category B: the appliance is only switched on when at least one occupant is present; the appliance stays on for a pre-programmed amount of time and switches itself off independently of occupant presence (e.g. washing-machine);

- Category C: the appliance is switched on and off by an occupant (e.g. shower, television ${ }^{9}$ );

- Category D: this accounts for the use of "miscellaneous" appliances (e.g. mobile phone chargers; video games consoles; hair-dryers, etc.) which are used occasionally and/or have relatively small power/water consumptions. Such minor individual appliances are not individually modelled. Rather, the cumulative consumption of these appliances, which are thus effectively grouped together, is represented by a randomly fluctuating positive value (within user specified limits).

At each hourly time step of the Solver, a sub-hourly loop runs through each instance of each appliance type. In order to calculate the power and/or water consumption of the appliance a loop first checks what category this appliance type belongs to and then (for Category B and $\mathrm{C}$ devices) decides randomly whether it is switched on according to a series of rules:

- if there is more than one appliance of a given type, a single occupant will use at most one appliance of this type;

- a random number is generated and compared to a switch-on probability profile to determine whether it is switched on;

\footnotetext{
${ }^{8}$ In theory, power consumed by a fridge will vary sinusoidally during the year with the internal temperature, but may temporarily increase when a door is opened to store or remove food. It also cycles i.e. switches on/off rather than operates continuously. However for the purposes of SUNtool these fluctuations will be relatively small and occur over a short time period (much shortly than the hourly time step of the overall Solver), so that they are ignored.

${ }^{9}$ Shared appliances such as cookers which are subject to communal use are handled slightly differently.
} 
- the duration of use is selected randomly from an exponential distribution and a countdown timer is started;

- for appliances which have a varying electrical power demand, the corresponding demand at the present time step is determined from a Gaussian distribution;

- for category $\mathrm{C}$ appliances, the appliance is switched off (early) if the occupant using the appliance either leaves the zone or becomes "inactive" (or asleep) as reported by the residential occupancy model.

This appliance model has been validated using data acquired from the LESO-PB building in Switzerland as well as from measurements in individual apartments. Note that although we discuss electrical appliances above, a similar rationale is applied with respect to water appliances except that the output rate is different $\left(1 \mathrm{~s}^{-1}\right.$ as opposed to $\mathrm{J} \mathrm{s}^{-1}$ ). Furthermore, in some cases an appliance may consume both water and electricity.

Special appliances with major resource usage implications such as domestic saunas can be optionally specified by the user via the GUI iDefaults editor by entering the appropriate categorisation and attributes. "Associated" consumptions such as the washing of hands after going to the toilet can also be handled in an intelligent way.

\subsubsection{Waste (refuse)}

The reasons for modelling refuse production are twofold. Firstly, this is in itself an indicator of the sustainability of a neighbourhood. Secondly and perhaps more importantly, energy can potentially be derived from its incineration. The produced waste can also be sorted into that which is recyclable and/or reusable. Temporally fine results are not necessary to support these assessments. For our purposes then, a weekly production is given by: $w_{k}=w_{\text {tot }} * p_{k} * 7 / 365 *$ occ $_{\text {ave }}$, where $w_{\text {tot }}$ is total waste production, $p_{k}$ the proportion of waste of the $k$ th type and occ $_{\mathrm{ave}}$ is the average number of occupants present during the week. An element of randomness concerning rates of production is added, so that $w_{k^{\prime}}=w_{k} *(1+\Delta w)$, where $\Delta w$ is a normal variable with a mean of 0 and a standard deviation of 0.5 , but with limiting extremes of \pm 1 .

Values of $p_{k}$ are derived from published statistical data within the region of interest.

\subsection{Plant modelling}

The thermal/fresh air needs of building zones are satisfied (capacity permitting) by zone emitters. These zone emitters are connected to an energy centre (which may in turn be connected to mains supply grids), by circuits which distribute either a fluid (air or water) for heating and/or cooling or electricity (in the case of electrical emitters). Energy centres may be building-embedded or decentralised district centres (Fig. 5).

Energy centres contain one or more sources for the supply of heat and/or power. The available technologies are presented in Table 1. Distribution losses between the district centre and buildings are also considered, and these sources may be prioritised, for example biasing first the use of renewable energy technologies, then non-renewable high efficiency technologies and finally grid resources or conventional non-renewable technologies.

For a given building it is possible to specify the following HVAC (heating, ventilation and air-conditioning) systems and controls via the GUI (Table 2).

In certain cases different technologies can be linked together. For instance, an absorption chiller could be solar powered (via a solar thermal collector) or use waste heat from a CHP engine, which can then produce chilled water

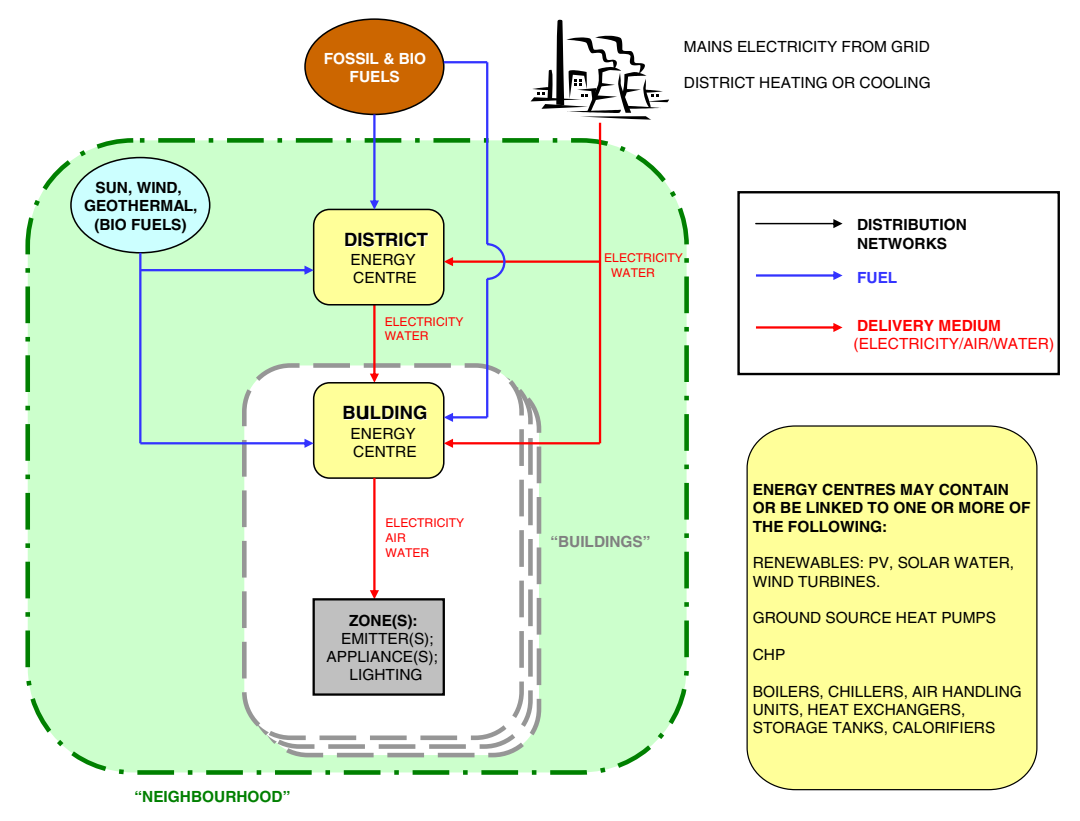

Fig. 5. Energy centres and distribution circuits (networks) in SUNtool. 
Table 1

SUNtool rules for connecting sources/generators to energy centres

\begin{tabular}{|c|c|c|c|c|c|c|c|c|c|}
\hline \multirow[t]{2}{*}{ Delivery medium (end use) } & \multicolumn{9}{|c|}{ Source/energy generator (not all listed) } \\
\hline & $\mathrm{DEC}$ & $\mathrm{CHP}$ & Boiler & Chiller & Heat pump & Solar thermal & PV & Wind turbine & Mains supply \\
\hline \multicolumn{10}{|l|}{ Building Energy Centre (BEC) } \\
\hline Hot water (space heating) & $\nabla$ & $\nabla$ & $\nabla$ & & $\nabla$ & $\nabla$ & & & $\nabla$ \\
\hline Heated air (ventilation/space heating) & $\nabla$ & $\nabla$ & $\nabla$ & & $\nabla$ & $\nabla$ & & & $\nabla$ \\
\hline Domestic hot water (washing) & $\nabla$ & $\nabla$ & $\nabla$ & & $\nabla$ & $\nabla$ & & & $\nabla$ \\
\hline Chilled water (space cooling) & $\nabla$ & & & $\nabla$ & $\nabla$ & & & & $\nabla$ \\
\hline Cooled air (ventilation/space cooling) & $\nabla$ & & & $\nabla$ & $\nabla$ & & & & $\nabla$ \\
\hline Electricity (lighting) & $\bar{\nabla}$ & $\nabla$ & & & & & $\nabla$ & $\nabla$ & $\bar{\nabla}$ \\
\hline Electricity (small power) & $\nabla$ & $\nabla$ & & & & & $\nabla$ & $\nabla$ & $\nabla$ \\
\hline \multicolumn{10}{|l|}{ District energy centre (DEC) } \\
\hline Hot water (space heating) & & $\nabla$ & $\nabla$ & & $\nabla$ & $\nabla$ & & & $\nabla$ \\
\hline Chilled water (space cooling) & & $\#$ & & $\nabla$ & $\nabla$ & $\#$ & & & $\bar{\nabla}$ \\
\hline Electricity (all power demands) & & $\nabla$ & & & & & $\nabla$ & $\nabla$ & $\bar{\nabla}$ \\
\hline
\end{tabular}

\#: If linked to e.g. an absorption chiller.

Table 2

Possible specifications of HVAC systems and controls at a building level

\begin{tabular}{ll}
\hline Per building per use & Options \\
\hline Heating system & Radiators; convectors (natural); underfloor heating; fan convector; fan coil unit (four-pipe); air (mechanical ventilation) \\
Cooling system & Chilled ceiling; chilled beam; fan coil unit (four-pipe); radiant panel (generic); air (comfort cooling) \\
Ventilation system & $\begin{array}{l}\text { Natural ventilation; mechanical ventilation (MV) [supply + extract]; mixed-mode for MV: fresh air requirement } \\
\text { (1/s/person) + heat recovery efficiency }\end{array}$ \\
$\begin{array}{l}\text { Environmental } \\
\text { conditions }\end{array}$ & $\begin{array}{l}\text { Fixed set-points for heating and cooling with plant on/off times and night-time setback } \\
\text { Dynamic set-points (that can be set on an hourly annual basis if so desired) via use of GUI profile/calendar }\end{array}$ \\
\hline
\end{tabular}

for an air-handling unit or an in-zone emitter (e.g. a fan coil unit or a chilled ceiling).

The Fuel/Plant Library contained in the GUI allows a user to choose from standard fuels and generic items of equipment (e.g. boilers, chillers, etc.) or to create bespoke fuels (e.g. a biomass source with a particular calorific value or $\mathrm{CO}_{2}$ emission factor $(\mathrm{g} / \mathrm{MJ})$, etc.) and plant items (e.g. based on manufacturers' performance data). These can then be freely connected to building or district energy centres as required. In this case the user may define a capacity, or this may be auto-sized (with the possibility of adjustment during a second simulation). As implied above, the predictor-corrector basis of the thermal solver leads to a drift of zone temperature from the associated set-point during periods of under supply.

Although a reasonably comprehensive plant modelling capability exists, albeit based on rather pragmatic performance curve based models, it should be noted that SUNtool is not intended to be a detailed design tool for energy (resource) management, e.g. testing and optimising control strategies and plant configurations. At present, SUNtool does not support energy storage.

\section{Interface design}

To ensure that the new interface would benefit from the best of current practice existing modelling software, within both 3D geometry and environmental modelling domains, were reviewed in some detail. Thus, whilst we have devel- oped a bespoke interface design tailored to environmental masterplanning, many specific design details have been borrowed from other tools. Examples include AutoCAD (www.autodesk.com), the 3D modelling tools Sketchup (www.sketchup.com), Rhino (www.rhino3d.com) and ArchiCAD (www.graphisoft.com) and the environmental modelling tools LT (www.carl.co.uk/lt-method.htm), Ecotect (www.squ1.com) and Tas (www.edsl.net).

\subsection{Problem definition}

On starting a new project, the user is first prompted to select the site location, using an image map of the globe. He or she may then either select the relevant weather file from the filtered list of those available or import a weather file.

If available, a national iDefault (intelligent default) dataset is automatically selected given the site location. These iDefaults minimise the need for user input (while allowing customisation). They handle input parameters such as: building fabric properties; internal partitioning/ zoning of space; building services systems; ownership of electrical and/or water consuming appliances; water and waste production; benchmarks, etc.

The national datasets ${ }^{10}$ actually contain a series of individual datasets based on historic construction standards - these vary from country to country and sometimes

\footnotetext{
${ }^{10}$ Note that users can copy and then edit these to create customised iDefaults datasets.
} 
regionally - depending on when new building regulations have been implemented.

For instance, when a user enters the year of construction when editing or attributing a building, this is automatically used as a key to select a default set of construction elements (walls, intermediate floors, windows, ground floor and roof) for the building depending on whether it is a residential or office building. However, users are free to edit or replace these constructions as they see fit. Since we have a simplified thermal model users are not required to build-up construction elements from individual layers and thicknesses (e.g. brick, air-gap, insulation, plasterboard) as this would impose a significant input burden, but only to describe high-level characteristics of the specific construction type they want to model (such as thickness, $U$-value and whether it is heavy, medium or lightweight).

\subsection{Drawing and attribution}

The GUI has a powerful 3-D modelling tool which allows users to rapidly create simple building geometries, to layer and group buildings and to visualise the masterplan (including the ability to run shadow animations for different times of the year).

The plan layout of the masterplan can be sketched in a 2-D plan window using native drawing tools. It is also possible for a user to import (to a separate layer) and scale a 2-D AutoCAD DXF or DWG and/or a bitmap image, as a basis for tracing the proposed building footprints. These footprints may then be extruded into 3-D entities using the 3-D window, where further drawing options (e.g. copying; cloning; creation of rectangular and polar arrays; pitched roofs, etc.) are possible. The user can also enter details on a District Energy Centre (DEC) by clicking on a "Plant" tab and connecting generic and/or custom plant items to appropriate distribution circuits.

Double clicking on an individual building allows detailed attribution of the building and editing of key property dialogues. By using a 'Matching' facility, the attribution changes may be automatically copied to other (related) buildings. Single clicking on an individual surface allows the properties of that individual part of a façade or roof to be edited independently via the "Surface Params" property dialogue, e.g. a user may want to assign different glazing ratios to different facades and so on.

Note that the user does not have to carry out any editing unless they wish to do so - basic attribution is automatically carried out on creation of a 3-D entity (building) using the selected iDefaults dataset. In this way the user can very quickly study the environmental implications of key early masterplanning issues such as the positioning and massing of buildings.

\subsection{Simulation and analysis}

Using the corresponding tab, simulations may be performed for either the set of buildings defined, or for some selected sub-set. The user may also choose the simulation period and the parameters to solve for. For example, rather than a fully integrated simulation, this user may wish firstly to solve for surface irradiation to assist with positioning $\mathrm{PV} / \mathrm{SWH}$ panels.

Batches of simulations may also be defined which will then run sequentially. Elements within these batches may be either simple single simulations for the defined period or parametric. In this latter case, the user is invited to select the parametric variables (only surface attributes are currently available for this facility) and define the lower limit together with the increment by which this variable is stepped to the upper limit.

Although presently a little limited, it is expected that this facility will be extended over time to include other variables, as well as multivariate analysis. Computational optimisation methods (such as genetic algorithms) may likewise be explored.

When a simulation is complete, a table summarising key environmental performance indicators for the modelled masterplan (overall and by building/group) is automatically displayed together with a series of standard graphs summarising the site's performance.

SUNtool also outputs fully interactive 3-D falsecolour plots and 2-D graphs. In the former case, building surfaces (relating to the zones that they envelope) may be coloured according to e.g. incident solar irradiation and heating, ventilation, cooling or lighting energy consumption; the chosen time period (hourly, monthly, annually).

\subsubsection{Runtime test}

As noted in Section 1, one of the key aims of SUNtool was that, as well as being quick and intuitive to learn and use, it should produce results of reasonable accuracy with reasonable speed. Now individual models have in general been individually verified and anecdotal comparisons with aggregate performance measurements for whole sites are also encouraging. Here then, we address simulation speed. For this we use two simple scenarios. The first, is comprised of four-bedroom detached residential houses (based on an $8 \mathrm{~m}$ by $8 \mathrm{~m}$ square footprint) consisting of two storeys and an unoccupied pitched roof. These are each represented by one zone. The second is an array of seven storey mixed use buildings, with offices at street level and residential flats above, so that each is represented by two zones. For both scenarios only building energy centres accommodating standard HVAC plant of infinite capacity are defined.

Annual simulations for different sized selections of these arrays of buildings were run on a single PC workstation with a single AMD AthlonTM XP 2200+ CPU (clockspeed $1.8 \mathrm{GHz}$ ) and $1 \mathrm{~GB}$ of RAM, running Microsoft Windows XP Professional (Version 5.1.2600 Service Pack 2 Build 2600). For reasons of consistency stochastic models were disengaged.

From Fig. 6 it is clear that, except for small initial preprocessing differences, the simulation time increases line- 


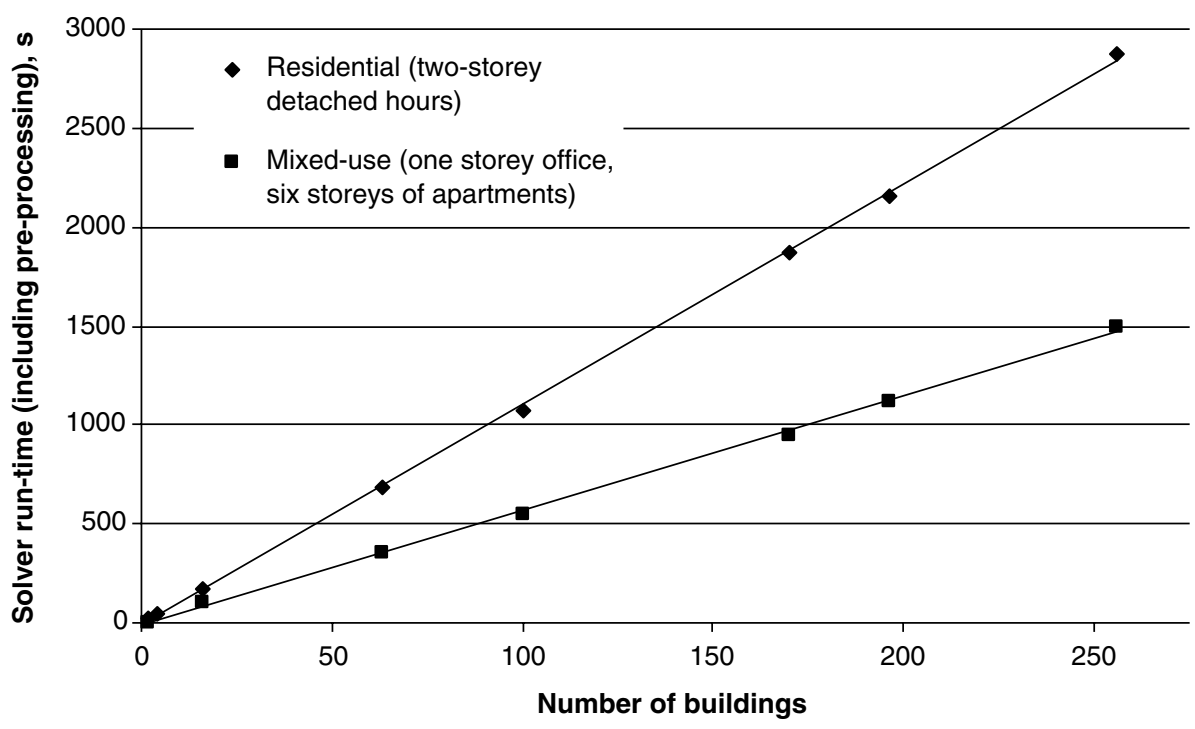

Fig. 6. Sensitivity analysis of solver run time as a function of number of buildings.

arly with the number of zones. For single zoned buildings, SUNtool solves for 100 buildings in $\sim 9 \mathrm{~m} 30 \mathrm{~s}$. For double zone buildings this increases to $\sim 18 \mathrm{~m} 30 \mathrm{~s}$. This is in the same range as a performance target that was established at the outset of the project: simulation of 100 buildings within $15 \mathrm{~m}$ ! Note that there is no intrinsic limit to the number of buildings modelled in SUNtool, except for that imposed by the time required to describe them or by the computing facilities available.

\section{Application}

By way of demonstration we have applied SUNtool to examine some initial relationships between building performance and the urban context and also to the analysis of building integrated photovoltaic panels to reduce net (electrical) energy consumption.

\subsection{Urban environmental planning guidelines}

A range of guidelines for the layout of buildings have been proposed in the past for the layout of buildings on urban sites to optimise the exploitation of available ambient energy resources whilst maintaining comfortable environments (see e.g. Littlefair, 1991; Littlefair et al., 2000). However, these guidelines tend to be based on highly simplified analyses - for example based on ensuring that obstruction altitudes are below some limit (such as the peak solar altitude during the winter solstice). Some simplified design tools, such as the LT Method, have attempted to take this analysis further; relating urban geometric parameters to energy consumption for heating, lighting and cooling buildings (Baker and Steemers, 1994). Indeed Robinson (2002) has demonstrated the application of a parametric front end to this model to derive 3D surface plots relating energy use to façade design and urban overshading. However, this is based on a steady-state ther- mal model of a single room, informed by a rather crude model of daylight and solar radiation transmission through windows. Such limitations are not present in SUNtool. Indeed, as far as we are aware SUNtool is the first integrated dynamic model which facilitates physically rigorous analyses of relationships between urban form and building energy consumption, from which to derive sound urban planning guidelines. In this section we present a short illustrative example of such an application.

For a hypothetical project located in Athens (Greece), we were interested to study and optimise building energy consumption as a function of façade glazing ratio and urban street canyon proportions - this latter expressed by height to width ratio $(h / w)$. As with the runtime test above, by creating an array of buildings (with the appropriate spacings between them and with facades composed of the appropriate glazing ratios) we can obtain the required set of results from a single SUNtool simulation.

From Fig. 7 we observe the expected trend that with less transmitted solar energy but the same fabric heat losses, heating energy consumption increases with $h / w$. Furthermore, the inverse trend is accentuated as glazing ratio increases. With reduced internal illuminances, lighting energy consumption also increases with $h / w$. There is a noticeable gradient change in the curves around $h / w=1$, as daylight sensors at the rear of rooms on lower floors have no sky view - which in fact is also evident in the shapes of heating and cooling curves, due to the associated heat gains. The cooling energy curve has the opposite trend, so that, the total carbon emissions have a dampened relationship with $h / w$. Nevertheless we can observe, for the kind of residential development studied here (in Athens), that south facing façades should be best designed with a glazing ratio below $30 \%$ and located in canyons of $h / w$ less than 1 . However, it is likely that a higher glazing ratio would be preferable if blinds were used to effectively reduce excess solar heat gains. 

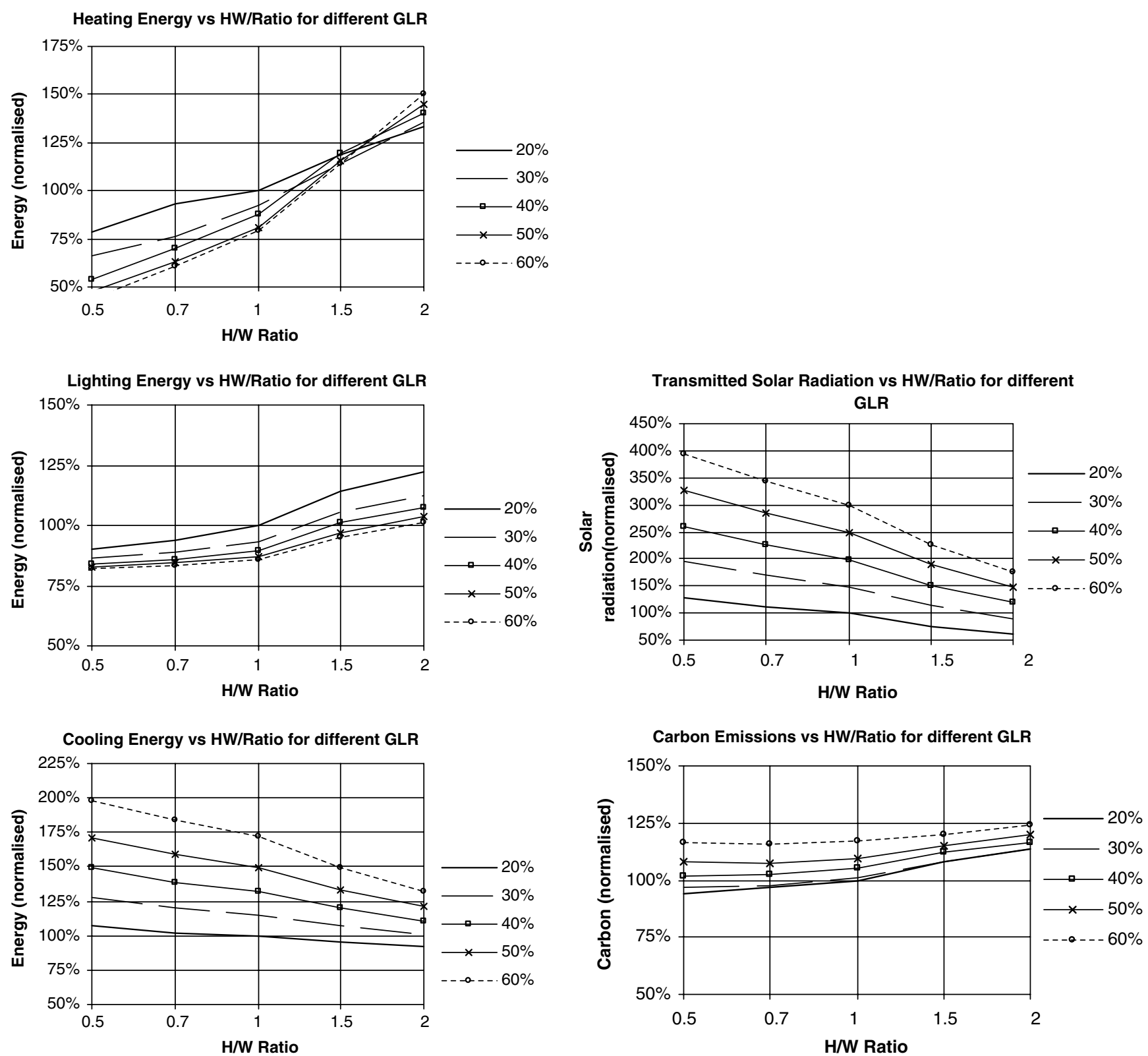

Fig. 7. Energy use (left), transmitted solar irradiation and Carbon emissions (right), normalised with respect to $h / w=1$ and a $20 \%$ glazing ratio.

\subsection{Case study}

Based again in Athens, this case study has examined relationships between building and urban form and energy consumption and has also studied the use of a range of renewable energy technologies to minimise net energy consumption (fossil fuel consumption). Fig. 8 shows the layout of the site - the Olympic Village.

Based purely on geometric considerations it is clear that, on an annual basis, collectors should either be horizontal or tilted less than $45^{\circ}$ and oriented within around $60^{\circ}$ of south (Fig. 9). Using SUNtool, the total electrical energy consumption, expressed on a per capita basis, has been predicted at $1.37 \mathrm{MWh} / \mathrm{m}^{2}$. On this basis, for a similar range of collector positions to those noted above, a per capita surface area of less then $5 \mathrm{~m}^{2}$ is required to match annual demand.

However, in the absence of demand for mechanical cooling beyond the summer months, there is frequently an excess of production, so that some $49 \%$ of energy produced is exported to the grid (Fig. 10) ${ }^{11}$ which, depending upon subsidisation schemes, may not be economically attractive. In reducing the installed area to just $1 \mathrm{~m}^{2} /$ person, some $84 \%$ of the energy produced is used directly on the site, but only one-fifth of the electrical energy needs are satisfied - suggesting the use of a complementary technology to further reduce net emissions.

\footnotetext{
${ }^{11}$ For simplicity, stochastic models have been disengaged in this study (deterministic profiles only are used).
} 

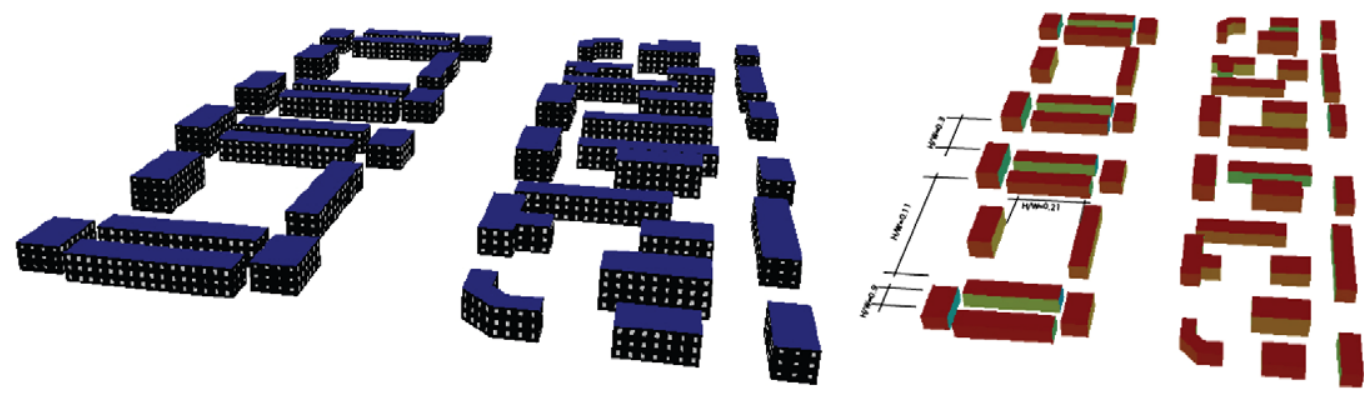

Fig. 8. Layout of case study site (left), with facades falsecoloured according to incident annual solar irradiation (right).
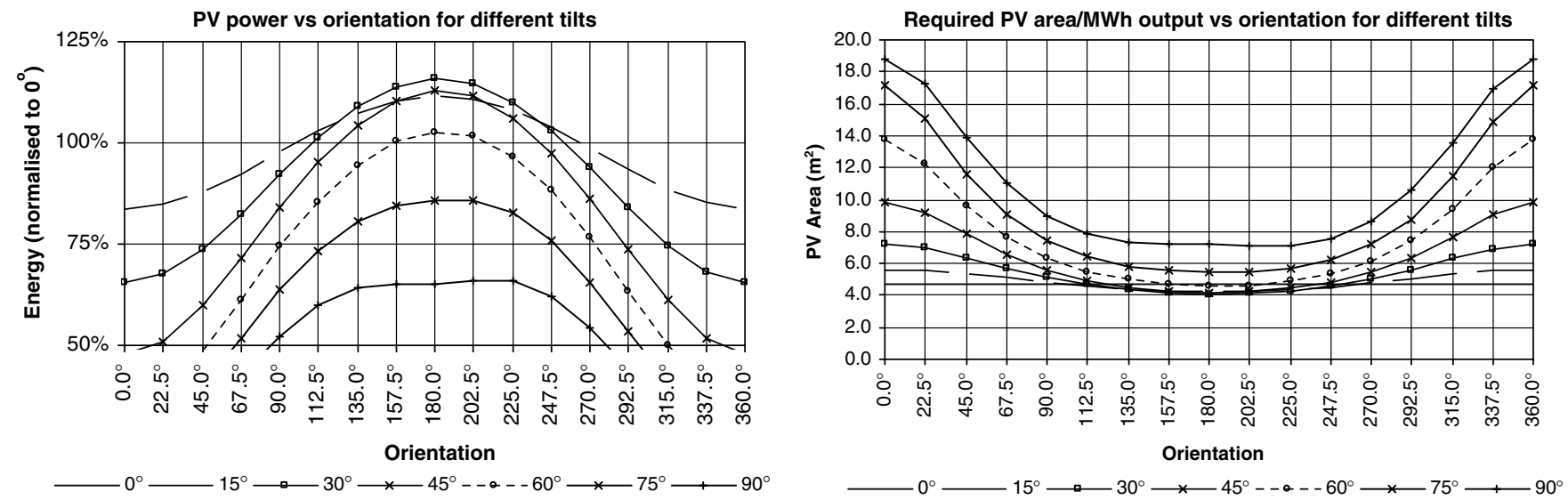

Fig. 9. Annual energy production relative to that of a horizontal collector (left) and required installed area to completely offset demand (right) of PV as a function of collector tilt and orientation.

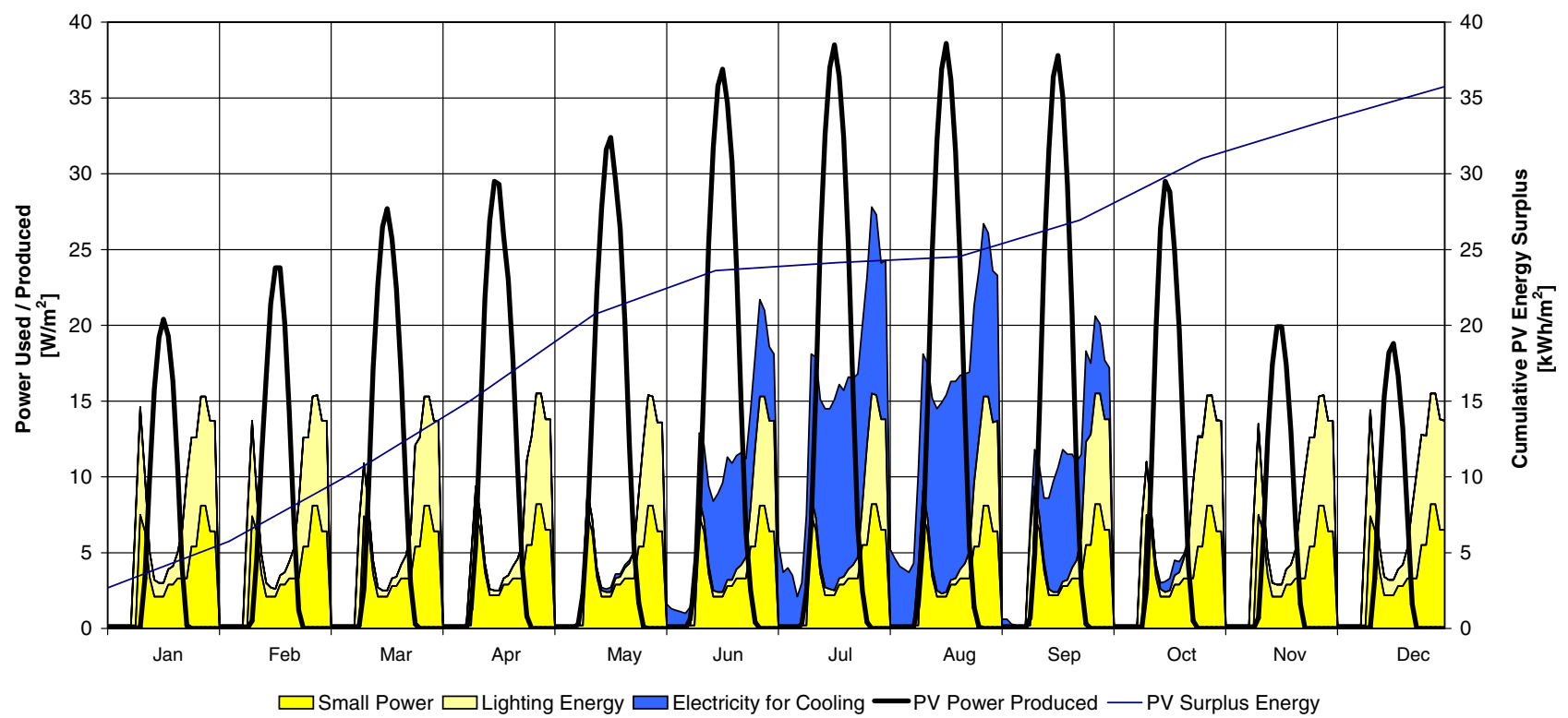

Fig. 10. Monthly mean daily profiles of end use electrical power consumption (with stochastic models disabled), PV energy production and cumulative excess of energy production.

\section{Discussion}

SUNtool represents a significant step forward in environmental modelling for the design and optimisation of sustainable urban neighbourhoods. Nevertheless, SUNtool is not a panacea. There are some significant limitations.

The modelling of building related resource flows is rather complete, but there are several gaps in this capability. 
Whilst the stochastic use of water appliances in buildings is modelled, the treatment of their output of grey (for recycling/reuse) and black (digestion and associated gas combustion) water, is not. Likewise the harvesting, treatment and storage of rainwater and the consumption of water (to balance evapotranspiration losses) for irrigation is not modelled. Furthermore, synergetic energy exchanges between buildings (heat rejection from one as a source for another) are not yet modelled, neither is the digestion of human waste to produce biogas, or the incineration of collected refuse. The production of energy from the range of renewable energy technologies is also not currently supported, though the majority of currently viable technologies are modelled. These however are relatively trivial processes to model and so they could be readily incorporated.

As discussed earlier, the demands for energy in buildings is influenced by the local thermal microclimate. However, due to the complex nature of the problem this is currently not represented. Research to find a good balance between modelling complexity and accuracy for the purposes of energy modelling is needed in this respect.

Non-building related energy exchanges include streetlighting and transport. Streetlights can be represented using a global iDefaults facility "Associated Modelling", but no attempt is made to model transport. Whilst is it fully recognised that transport has very important implications for urban sustainability, this deliberate decision is based on an incompatibility in scale between a neighbourhood, say of one to several hectares, and a town or city (or part of a city) in which road and public transport networks may be significantly modified.

Other key gaps in modelling capability include:

- Capital and life cycle cost analysis, including the energy and environmental cost of constructional materials.

- Modelling of uncertainties in input parameters, particularly at the early stages of masterplanning new neighbourhoods.

Finally, it would be useful to define some quantitative measure of environmental sustainability which harmonises in an objective way the various flows of energy and matter in an urban neighbourhood to better guide the optimisation process. Related to this, it would also be useful to use some form of computational procedure to search the available parameter space for optimal environmental sustainable solutions for both new and refurbishment projects.

Nevertheless, as far as we are aware, SUNtool does at present provide the most complete basis available for urban energy optimisation. Efforts have been made to confirm the validity of each individual model and anecdotal comparisons with a Czech apartment block suggest that the combination of these models provides satisfactory predictions $^{12}$ - but this is subject to the quality of model cali-

\footnotetext{
${ }^{12}$ Detailed work on whole model validation has not been carried out, due to the difficulty of isolating causes for predictive errors, should these exist.
}

bration exercise - which may be achieved either by customising iDefaults relating to external features (e.g. streetlights), building characteristics, plant systems and occupant characteristics, etc. or by adapting the values subsequently attributed to individual buildings/surfaces.

\section{Conclusions}

SUNtool is the first of a new genre of simulation tool, developed to support urban designers to optimise the environmentally sustainability of their masterplanning proposals. From the outset of its development, the aims were to develop a quick and easy to use interface to describe the factors which influence the dynamic demand (mainly for buildings) and supply of energy, water and waste. An integrated solver should simulate the flows of these resources (energy and matter) in a way that is sensitive to the urban microclimate, to human behaviour, to synergies between buildings and resources and finally to the presence of district resource management centres. Realising each of these objectives, has led to the following innovations:

- A facility to rapidly attribute buildings using intelligent defaults (iDefaults) and methods for copying and cloning buildings and their attributions.

- A new integrated radiation model for simulating shortwave and longwave radiation exchange and interior daylight, fully accounting for external obstructions and variable sky radiance/luminance distributions.

- A new reduced thermal model and its application to solve for multi-zone buildings.

- A family of stochastic models, predicting occupant presence and their related interactions with windows, shading devices, lights and electrical and water appliances as well as associated refuse production.

- Automatic zoning of internal spaces, according to internal use and external views (influencing the incident irradiation).

- A family of 'energy source' models as children of 'energy centres' which may be building embedded or site wide, facilitating complementary connections between these sources and accounting for distribution losses from district centres to the distributed buildings.

- The solver simulates for more than 100 monozone buildings in fewer than $10 \mathrm{~min}$.

In achieving these objectives SUNtool is uniquely capable of producing urban energy planning guidelines taking into account the set of energy implications of the urban context on building's energy consumption. It is also uniquely placed to support the (environmentally) sustainable masterplanning process - from defining building layouts, their forms and façades, through to the energy systems supplying them.

For further information see www.suntool.net, from which a public version of SUNtool will shortly be freely available for download. 


\section{References}

Baker, N.V., Steemers, K.A., 1994. LT Method Version 2.0. Cambridge Architectural Research Ltd, Cambridge, UK.

Clarke, J.A., 2001. Energy Simulation in Building Design, second ed. Butterworth Heinemann, Oxford.

Déqué, F., Olivier, F., Poblador, A.M., 2000. Grey boxes used to represent buildings with a minimum number of geometric and thermal parameters. Energy and Buildings 31 (1), 29-35.

Elnahas, M.M., Williamson, T.J., 1997. An improvement of the CTTC model for predicting urban air temperatures. Energy and Buildings 25, $41-49$.

Fanger, P.O., 1988. Introduction of the olf and decipol units to quantify air pollution perceived by human indoors and outdoors. Energy \& Buildings, 12.

Graves, H., Watkins, R., Westbury, P., Littlefair, P., 2001. Cooling building in London: overcoming the heat island. BRE Report BR 431. CRC Ltd: London.

Herkel, S., Knapp, U., Pfafferott, J., A preliminary model of user behavious regarding the manual control of windows in office buildings. In: Proc. 9th Int. IBPSA Conf. BS2005, Montréal, pp. 403-410.

Judkoff, R., Neymark, J., 1995. International energy agency building energy simulation test (BETSEST) and diagnostic model. US Department of Energy, National Renewable Energy Laboratory: Golden, CO, p. 296 (+Disk).

Kusaka, H., Kondo, H., Kikegawa, Y., Kimura, F., 2001. A simple singlelayer urban canopy model for atmospheric models: comparison with multi-layer and SLAB models. Boundary Layer Meteorology 101, 329-358.

Littlefair, P.J., 1991. Site layout and planning for daylight and sunlight: a guide to good practice. BRE Report 209. BRE Bookshop: UK.

Littlefair, P.J., Santamouris, M., Alvarez, S., Dupagne, A., Hall, D., Teller, J., Coronel, J.F., Papanikolaou, N., 2000. Environmental site layout planning: solar access, microclimate and passive cooling in urban areas. BRE Report 380. CRC Limited: UK.

Martilli, A., Clappier, A., Rotach, M.W., 2002a. An urban surfaces exchange parameterisation for mesoscale models. Boundary Layer Meteorology 104, 261-304.

Masson, V., 2000. A physically-based scheme for the urban energy budget in atmospheric models. Boundary Layer Meteorology 94, 357-397.

Moore, B.C., 1981. Principal component analysis in linear system: controllability, observability and model reduction. IEE Transactions on Automatic Control AC 26, 17-32.

Nicol, J.F., 2001. Characterising occupant behaviour in buildings: towards a stochastic model of occupant use of windows, lights, blinds, heaters and fans. In: Proc. 7th Int. IBPSA Conf. BS2001, Rio, pp. 1073-1078.
Oke, T.R., 1973. City size and the urban heat island. Atmospheric Environment (7), 769-779.

Oke, T.R., 1988. Street design and urban canopy layer climate. Energy and Buildings (11), 103-113.

Page, J., Robinson, D., Morel, N., Scartezzini, J.-L., A generalised stochastic model for the prediction of occupant presence. Energy and Buildings, in press, doi:10.1016/j.enbuild.2007.01.018.

Reinhart, C.F., 2004. Lightswitch-2002: a model for manual and automated control of electric lighting and blinds. Solar Energy 77 (1), 15-28.

Robinson, D., 2002. Concept environmental design tools: some new additions to the family. In: Proc. ISES Summer Academy, Johannesburg, 2002.

Robinson, D., 2006a. Trends and research needs in energy and comfort prediction. In: Proc. Comfort and Energy Use in Buildings, Windsor, UK, April 2006. <http://nceub.org.uk/uploads/Robinson.pdf $>$.

Robinson, D., Stone, A., 2004. Solar radiation modelling in the urban context. Solar Energy 3 (77), 295-309.

Robinson, D., Stone, A., 2005a. A simplified radiosity algorithm for general urban radiation exchange. Building Services Engineering Research and Technology 26 (4), 271-284.

Robinson, D., Stone, A., 2005b. Holistic radiation modelling with a fast simplified radiosity algorithm. In: Proc. Ninth Int. IBPSA Conf., Building Simulation 2005, Montreal, Canada.

Robinson, D., Stone, A., 2006. Internal illumination prediction based on a simplified radiosity algorithm. Solar Energy 80 (3), 260-267.

Robinson, D., Stankovic, S., Morel, N., Deque, F., Rylatt, M., Kabele, K., Manolakaki, E., Nieminen, J., 2003. Integrated resource flow modelling of urban neighbourhoods: Project SUNtool. In: Proc. Building Simulation 2003, Eindhoven.

Steemers, K.A., 1992. Energy in buildings: the urban context. Unpublished $\mathrm{Ph} . \mathrm{D}$. Thesis. University of Cambridge.

Swaid, H., 1988. The physical basis for the analytical-experimental model for predicting the urban climate. D.Sc. Thesis, Technion, Israel Institute of Technology.

Swaid, H., Hoffman, M.E., 1989. The prediction of impervious ground surface temperature by the Surface Thermal Time Constant (STTC) model. Energy and Buildings 13, 149-157.

Swaid, H., Hoffman, M.E., 1990a. Prediction of urban air temperature variations using the analytical CTTC model. Energy and Buildings 14, 313-324.

Swaid, H., Hoffman, M.E., 1990b. Climatic impacts of urban design features for high and mid latitude cities. Energy and Buildings 14, 325-336.

Ward Larsen, G., Shakespeare, R., 1997. Rendering with Radiance - The Art and Science of Lighting Visualisation. Morgan Kauffmann, San Francisco. 